\title{
Peertechz
}
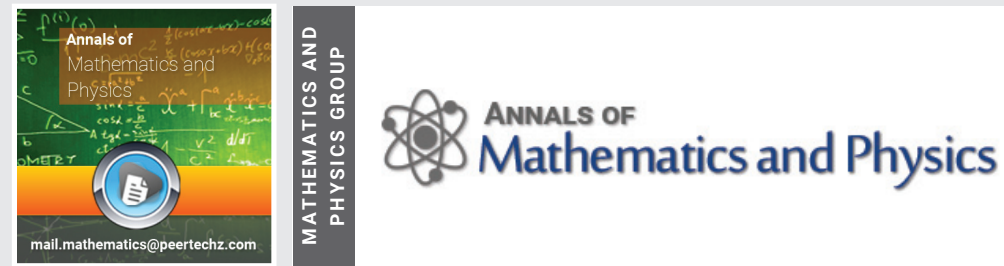

Research Article

\section{An astrobiological theorem}

\author{
David A Steinberg* \\ Fiddletown Institute, 15111 Tyler Road, Fiddletown, CA 95629, USA
}

Received: 25 April, 2020

Accepted: 16 October, 2020

Published: 17 October, 2020

*Corresponding author: David A Steinberg, Fiddletown Institute, 15111 Tyler Road, Fiddletown, CA 95629 USA, Tel: 209-245-6178, 209-765-8999;

E-mail: dasteinberg@fiddletownca.net

https://www.peertechz.com

\section{Abstract}

The structure of the human brain reflects multifarious random influences of terrestrial and phylogenetic history, yet the higher mental functions correlated with this unique cerebral neurophysiology are generally assumed to embody universals common to intelligences independent of biological substrate. This assumption is deeply embedded in scientific and popular cultures. However, this idea has not been explicitly investigated. The present study proves that any sufficiently advanced organism of non-zero, finite volume (with boundary) must have a 'natural' logic equivalent to Sentential (propositional) Calculus (SC). This commonalty arises from the essential transduction from external to internal milieu that must occur at any organism's boundary surface. This transduction encodes SC in sensory data and the proof demonstrates that any internal inductive construct-including mathematics and physics-inherits this logical bias. The topological origin of deductive logic not only demonstrates a universal commonality subject to very weak constraints, but also demonstrates a surprising biological origin of foundational principles in mathematics and physics.

\section{Introduction and axioms}

The demonstration of a commonalty of logical structures in arbitrary organisms under weak topological constraints is in two parts. The first is a proof that the process of transduction from external to internal milieu encodes sentential calculus (SC) in sensory data. It is well known that $\mathrm{SC}$ is quite robust, and appears in many guises-probably most familiar in switching circuits, as shown by Claude Shannon in his 1937 Master's thesis [1]. Therefore, the proof that sensory transduction is equivalent to $\mathrm{SC}$ - though necessary, and occupying the bulk of the paperis not particularly surprising. The second part-containing the astrobiological significance of this equivalence-arises from a demonstration of the persistence of the logical bias of SC in all inductive constructs of an organism that form its internal model of the external world. The first axiom of this analysis is that the external world can be known only as such an internally constructed model. The second axiom is that the internal model is ordinally and inductively derived-i.e., each level of increasing complexity results from an equivalence identified in a preceding stage. However, particular details of the model's construction - and its many additional consequences [2-4]are not directly relevant to the current proof. Given the two axioms, the theorem demonstrates that topological exigencies impose a commonalty of logic shared by any physical organism.

Though the conclusion of this proof is consistent with the orthodox assumption of a commonalty amongst diverse intelligences, this agreement is fortuitous and actually arises by correcting an error in the common view. Though rarely, if ever discussed, natural scientists implicitly consider the external world and its internal model to be isomorphicinformally, interchangeable or equivalent. Therefore, an alternate intelligence observing the same universe should share the more abstract (and non-idiosyncratic) elements of the internal model, e.g., mathematics and physics, and it has been traditionally thought that effective communication with such an organism would proceed by use of these disciplines. However, this fundamental assumption-i.e., the isomorphism of external world and internal model-is, in fact, incorrect. The functional relationship between the external and internal worlds is actually the composition of two maps-decomposition (discretization/transduction) and reconstruction. Since the former of these has no inverse, an isomorphism cannot exist, and the external universe and its internal model are incommensurable. Therefore, any extrapolations from human perspectives cannot be assumed, but must be carefully verified. Subject to weak topological constraints, this proof demonstrates that there is a commonalty underlying any organisms' internal model of the universe, but its origin is entirely distinct from that currently supposed, and as a result, the proof has consequences for the sciences of mathematics and physics that have not been recognized.

The topological origin of SC has other consequences independent of the existence of extraterrestrial, or alternate, 
intelligence. The theorem provides new perspectives on classic problems in natural science and mathematics, in particular, the nature of mathematical forms, and the utility of mathematics in physics. The biological origin of these perspectives identifies an unexpected and interesting connection between fundamental sciences.

The proof originates in the observation that any organism can know the external world solely as an internally constructed model. A prerequisite for such a reconstruction is a process of transduction at the interface between external and internal worlds - a boundary that must exist in any physical organism. In humans, the sensory organs perform this function. The transduction apparatus converts a small portion of the multifarious ambient information into a common internal form (e.g., bioelectric impulses) from which the external world is internally reconstructed. The external world must be discretized, selectively filtered, and internally reassembled before it is knowable, and this process has consequences that can be formally analyzed.

\section{Overview}

This investigation first generalizes aspects of human sensory transduction that apply to any organism. The external stimulation of an arbitrary organism becomes a pattern of activation of its transducing units. Some complex patterns of stimulation will be identical to less complex patterns, while others may not be so reducible. Analysis of the equivalence of activation patterns by formal rules that characterize the transduction process results in a calculus of stimuli.

After interpreting its symbols, the calculus is proved to be equivalent to the sentential calculus of deductive logic. This shows that transduction creates a logical structure that is embedded at the first stage of perception of the external world. It is then demonstrated that any inductive construct internally created from sensory data must preserve the calculus of stimuli, and thereby preserve the logical bias established by sensory transduction. An astrobiological theorem results from the essential nature of transduction for any organism whose structure satisfies very weak topological constraints.

The proof, then, consists of the following steps: first, abstract principles common to all systems of sensory transduction are isolated; second, a calculus of stimuli is presented that identifies the equivalence of different activation patterns; third, the symbols of the calculus are interpreted; fourth, it is proven that under this interpretation the calculus of stimuli is equivalent to SC; fifth, it is proven that any inductive constructs created from sensory data retain the logical bias of SC; finally, it is concluded that any sentient physical organism must have a system of logic equivalent to SC.

\section{A generalized system of sensation}

Sensory transduction is the discrete filtering of continuous and diverse external information into a common internal mode from which the external world can be reconstructed. The filtering is accomplished by activation of a set of reactive elements of an organism in response to specific external stimuli. Activation of one element may result in the inhibition or activation of other elements, by its action alone or by summation with other elements. In order to account for interactions of basic transducing elements, a transducing unit will be defined as a collection of interacting elements. Any element may be its own unit, as well as part of many other units. The specific details of the structure of transducing units, however, are not germane to the present analysis. The calculus depends on only two fundamental aspects of transduction-1. activation of a transducing unit can be considered a binary process, wherein the unit is either active or not, and 2 . different patterns of activation are equivalent. Note that any logical constraints imposed by the binary case apply equally to a system with graded responses of the transducing units since this circumstance is described by the conjunction of the propositions (unit ' $a$ ' is active) \& (unit ' $a$ ' has output ' $x$ ').

\section{A calculus of stimuli}

In the following, set-theoretic symbols are used for clarity and convenience, but the use is mereologic - the symbols refer to actual physical objects, and parts of these objects. Let $\mathrm{A}^{*}$ $=\left\{a_{i}\right\}_{i \in \omega}$ be the transducing units of an arbitrary organism. For each finite organism there will be an ' $n$ ' such that $a^{*}{ }_{i}=$ $\emptyset$ for $\mathrm{i}>\mathrm{n}$. Let $\wp\left(\mathrm{A}^{*}\right)=$ power set of $\mathrm{A}^{*}$. Let activation of a transducing unit $\mathrm{a}^{*}{ }_{\mathrm{i}}$ be denoted by ai. Note that if $\mathrm{a}^{*}{ }_{\mathrm{i}}=\emptyset$, then $\mathrm{a}_{\mathrm{i}}=\emptyset$.

Activation of a transducing unit is evidenced by output from the unit. Let the following two operations be defined:

1. $a_{i} a_{j}-$ concatenation, simultaneously activating $a^{*}{ }_{i}$ and $a^{*}$, concatenation is commutative (comm). In the set $\wp\left(\mathrm{A}^{*}\right)$, this means activating the element $\left(\mathrm{a}^{*}{ }_{\mathrm{i}}\right) \cup\left(\mathrm{a}^{*}{ }_{\mathrm{j}}\right)$ where the union is in $\wp\left(\mathrm{A}^{*}\right)$.

2. $\left[\mathrm{a}_{\mathrm{i}}\right]$ - complement, activating $\mathrm{p}^{*} \in \wp\left(\mathrm{A}^{*}\right)$ such that $\mathrm{p}^{*}$ $=A^{*} \backslash a^{*}{ }_{i}$ with ' $=$ ' meaning 'the same element as' in $\wp\left(\mathrm{A}^{*}\right)$.

A stimulus can now be defined as follows:
1. $a_{i}$ is a stimulus
2. $\left[a_{i}\right]$ is a stimulus
3. $a_{i} a_{j}$ is a stimulus

4. any finite application of $1-3$ is a stimulus

Let $a, b, c, \ldots$, etc. represent stimuli generated by the above rules.

With '=' meaning 'the same pattern of activation as', with ' $A$ ' representing activation of the entire set $A^{*}$, and with $\emptyset$ representing absence of activation of any element of $A^{*}$, the following primitive equalities, which characterize transduction, are easily verified as consequences of the definitions of concatenation and complementation:

I. $\mathrm{a} a=\mathrm{a}$ 


$$
\begin{aligned}
& \text { II. }[[a]]=a \\
& \text { III. }[a] a=A,[[a] a]=\emptyset \\
& \text { IV. } A a=a A=A, \emptyset a=a \emptyset=a \\
& \text { V. }[a b] b=[a] b
\end{aligned}
$$

Activation of the entire set $A^{*}$ and the empty activation, $\emptyset$, cannot be obtained by a finite application of rules 1-3 above and therefore as stimuli, $A$ and $\emptyset$ are used as diminutives for the identities, $A \equiv[\mathrm{a}] \mathrm{a}$, and $\emptyset \equiv[[\mathrm{a}] \mathrm{a}]$.

Any stimulus can be reduced to an irreducible stimulus by the application of I-V above. Some stimuli are reducible to activation of the entire set $A^{*}$, and some are reducible to the empty activation, $\varnothing$. The collection of stimuli that reduce to the stimulus, A, by application of I-V, I will call STIMA. As examples, I will now reduce the following seven stimuli, the choice of which will be clear shortly:

1. [a] [b] a:

[a] [b] a $=[\mathrm{a}] \mathrm{a}[\mathrm{b}]$

comm

$[\mathrm{a}] \mathrm{a}[\mathrm{b}]=\mathrm{A}[\mathrm{b}]$

III.

$\mathrm{A}[\mathrm{b}]=\mathrm{A}$

IV.

2. $[[a][b] c][[a] b][a] c:$

$[[a][b] c][[a] b][a] c=[[a][b] c][b[a]][a] c$

comm

$[[a][b] c][b[a]][a] c=[[a][b] c][b][a] c$

$[[a][b] c][b][a] c=[[a][b] c][a][b] c$

\author{
V.
}

comm

let $d=[a][b] c$, then

[d] $\mathrm{d}=\mathrm{A}$

3. $[[[a]][b]][b] a:$

$[[[a]][b]][b] a=[a[b]][b] a$

$[\mathrm{a}[\mathrm{b}]][\mathrm{b}] \mathrm{a}=[\mathrm{a}[\mathrm{b}]] \mathrm{a}[\mathrm{b}]$

let $\mathrm{c}=\mathrm{a}[\mathrm{b}]$, then

$[\mathrm{c}] \mathrm{c}=\mathrm{A}$

4. $[[[a][b]]] a$, or $[[[a][b]]]$ b:

$[[[a][b]]] a=[a][b] a$

$[\mathrm{a}][\mathrm{b}] \mathrm{a}=[\mathrm{a}] \mathrm{a}[\mathrm{b}]$

$[\mathrm{a}] \mathrm{a}[\mathrm{b}]=\mathrm{A}[\mathrm{b}]$

$\mathrm{A}[\mathrm{b}]=\mathrm{A}$

5. $[[c] a][[c] b][c][[a][b]]:$

$[[c] a][[c] b][c][[a][b]]=[[c] a][b[c]][c][[a][b]]$

comm

$[[c] a][b[c]][c][[a][b]]=[[c] a][b][c][[a][b]]$

III. $[[c] a][b][c][[a][b]]=[a[c]][c][b][[a][b]]$

comm

$[\mathrm{a}[\mathrm{c}]][\mathrm{c}][\mathrm{b}][[\mathrm{a}][\mathrm{b}]]=[\mathrm{c}][\mathrm{a}][\mathrm{b}][[\mathrm{a}][\mathrm{b}]]$

V., comm

let $d=[a][b]$, then

$[c] d[d]=[c][d] d=[c] A$

comm, III.

[c] $\mathrm{A}=\mathrm{A}$

IV.

6. [a] a b, or [b] a b:

$$
\begin{aligned}
& \text { [a] } \mathrm{a} \mathrm{b}=\mathrm{A} \mathrm{b} \\
& \mathrm{A} \mathrm{b}=\mathrm{A}
\end{aligned}
$$

III.

IV.

7. $[[a] c][[b] c][a b] c:$

[[a] c] [[b] c] [a b] c = [[a] c] [[b] c] c [a b]

comm

[[a] c] [[b] c] c [a b] = [[a] c] [[b]] c [a b]

V.

$[[a] c] b c[a b]=[[a] c] c b[a b]$

II., comm

$[[a] c] c b[a b]=[[a]]$ c b $[a b]$

V.

$[[a]] c b[a b]=a c b[a b]$

II.

$\mathrm{a} \mathrm{c} \mathrm{b}[\mathrm{ab}]=[\mathrm{a} b] \mathrm{abc}$

comm

let $\mathrm{d}=\mathrm{ab}$, then

[d] d c $=$ A c

III.

$\mathrm{Ac}=\mathrm{A}$

IV.

\section{An interpretation of the calculus}

The symbols of the calculus of stimuli can be interpreted in the following manner:

1. let $\left\{a_{i}\right\}_{i \in \omega}$ be identified with the set of sentential variables $\left\{p_{i}\right\}_{i \in \omega}$

2. let concatenation be interpreted as the logical connective 'or', i.e., $a_{i} a_{j} \equiv p_{i} \vee p_{j}$ where ' $a_{i}$ ' is identified with ' $p_{i}$ ' and ' $a_{j}$ ' with ' $\mathrm{p}_{\mathrm{j}}$ '

3. let [ ] be interpreted as the logical connective 'not', i.e., $\left[\mathrm{a}_{\mathrm{i}}\right] \equiv \sim \mathrm{p}_{\mathrm{i}}$

then,

4. the logical connective 'and' becomes, $p_{i} \wedge p_{j} \equiv\left[\left[a_{i}\right][a j]\right]$

5. the logical connective 'imply' becomes $p_{i} \rightarrow p_{j} \equiv\left[a_{i}\right] a_{j}$

Note that for any realization, $\mathrm{R}$, of $\mathrm{SC}, \mathrm{R}\left(\mathrm{f}_{\mathrm{A}}\right)=\mathbf{1}$ and $\mathrm{R}(\mathrm{f} \emptyset)$ $=\mathbf{0}$, where $\mathrm{f}_{\mathrm{A}}$ and $\mathrm{f} \emptyset$ are the formulas of $\mathrm{SC}$ obtained from $\mathrm{A} \equiv$ [a] a, and $\emptyset \equiv[[\mathrm{a}] \mathrm{a}]$.

The common properties of the logical connectives are retained in this interpretation, and any stimulus can be translated into a formula and vice versa. It must be shown, however, that the translations are unambiguous, and, more 
importantly, that reduction of a stimulus preserves the realization of the translated formula.

\section{Equivalence of the calculus of stimuli and SC}

Lemma 1: Every stimulus translates to a unique formula of SC, and vice versa.

pf: The proof is by induction on the number of logical connectives and elementary operations.

1. Every formula translates to a unique stimulus.

a. sentential variables translate to atomic stimuli, $\mathrm{pi} \rightarrow \mathrm{ai}$

b. suppose that $\phi$ and $\psi$ translate uniquely to $\mathrm{a}$ and $\mathrm{b}$, then

$\phi \vee \psi$ and $\sim \phi$ also translate uniquely.

1. $\phi \vee \psi$ translates uniquely to $\mathbf{a} \mathbf{b}$

2. $\sim \phi$ translates uniquely to $[\mathrm{a}]$

2. Every stimulus translates to a unique formula.

a. atomic stimuli translate to sentential variables, ai $\rightarrow$ pi

b. suppose that a and $\mathrm{b}$ translate uniquely to $\phi$ and $\psi$, then $\mathrm{a} b$ and $[\mathrm{a}]$ also translate uniquely.

1. a b translates uniquely to $\phi \vee \psi$

2. [a] translates uniquely to $\sim \phi$

Lemma 2: Reduction of a stimulus, s, preserves the realization of $f_{s}$, the formula of SC derived from $s$.

pf: It must be shown that $R\left(f_{s}\right)=R\left(f_{s}{ }^{\prime}\right)$ if $s$ reduces to $s^{\prime}$ by application of rules I-V. The number of applications of I-V in the reduction of $s$ to $s^{\prime}$ is finite since $s$ is finite. If each reduction can be shown to preserve realization,

$\mathrm{R}\left(\mathrm{f}_{\mathrm{s}}\right)=\mathrm{R}\left(\mathrm{f}_{\mathrm{s} 1}\right)=\ldots=\mathrm{R}\left(\mathrm{f}_{\mathrm{sn}}\right)=\mathrm{R}\left(\mathrm{f}_{\mathrm{s}}{ }^{\prime}\right)$ and, therefore, $\mathrm{R}\left(\mathrm{f}_{\mathrm{s}}\right)=\mathrm{R}\left(\mathrm{f}_{\mathrm{s}}{ }^{\prime}\right)$.

It suffices to show both sides of the equality sign in I-V have the same realization. The symbols $\wedge$ and $\vee$ are the operations meet and join when connecting realizations, and Rc is the complement of R, both in the Boolean Algebra, 2.

$$
\begin{aligned}
& \text { I. } R\left(f_{a} \vee f_{a}\right)=R\left(f_{a}\right) \vee R\left(f_{a}\right)=R\left(f_{a}\right) \\
& \text { II. } R\left(\sim\left(\sim f_{a}\right)\right)=R\left(\sim f_{a}\right) c=R\left(f_{a}\right) c c=R\left(f_{a}\right) \\
& \text { III. } R\left(\sim f_{a} \vee f_{a}\right)=R\left(f_{a}\right) c \vee R\left(f_{a}\right)=1=R\left(f_{A}\right) \\
& \text { R( } \left.\sim\left(\sim f_{a} \vee f_{a}\right)\right)=R\left(\sim f_{a} \vee f_{a}\right) c=\left(R\left(f_{a}\right) c \vee R\left(f_{a}\right)\right) c=1 c=0=R(f \emptyset) \\
& \text { IV. } R\left(f_{A}\right) \vee R\left(f_{a}\right)=1 \vee R\left(f_{a}\right)=R\left(f_{a}\right) \vee 1=R\left(f_{a}\right) R(f \emptyset) \vee R\left(f_{a}\right)=0 \vee \\
& R\left(f_{a}\right)=R\left(f_{a}\right) \vee 0=R\left(f_{a}\right) \\
& \text { V. } R\left(\sim\left(f_{a} \vee f_{b}\right) \vee f_{b}\right)=R\left(\sim\left(f_{a} \vee f_{b}\right)\right) \vee R\left(f_{b}\right)=\left(R\left(\sim f_{a}\right) \wedge R\left(\sim f_{b}\right)\right) \vee \\
& R\left(f_{b}\right)=\left(R\left(\sim f_{a}\right) \vee R\left(f_{b}\right) \wedge\left(R\left(f_{b}\right)\right) c \vee R\left(f_{b}\right)\right)=\left(R\left(\sim f_{a}\right) 1 R\left(f_{b}\right)\right) \wedge \\
& 1=R\left(\sim f_{a}\right) \vee R\left(f_{b}\right)=R\left(\sim f_{a} \vee f_{b}\right)
\end{aligned}
$$

Lemma 3: If $\mathrm{R}$ is any realization of $\mathrm{SC}$, and if a stimulus, $\mathrm{s}$, reduces to $A$, then $R\left(f_{s}\right)=1$. pf. Let $\mathrm{s}^{\prime}=\mathrm{A}$ in Lemma 2 , then $\mathrm{R}\left(\mathrm{f}_{\mathrm{s}}\right)=\mathrm{R}\left(\mathrm{f}_{\mathrm{A}}\right)$. But, $\mathrm{R}\left(\mathrm{f}_{\mathrm{A}}\right)=\mathbf{1}$.

Therefore, $\mathrm{R}\left(\mathrm{f}_{\mathrm{s}}\right)=\mathbf{1}$.

The seven previous stimuli examples can now be translated as:
1. $\phi \rightarrow(\psi \rightarrow \phi)$
2. $[\phi \rightarrow(\psi \rightarrow \chi)] \rightarrow[(\phi \rightarrow \psi) \rightarrow(\phi \rightarrow \chi)]$
3. $(\sim \phi \rightarrow \sim \psi) \rightarrow(\psi \rightarrow \phi)$
4. $(\phi \wedge \psi) \rightarrow \phi, \quad \circ \rho(\phi \wedge \psi) \rightarrow \psi$
5. $[\chi \rightarrow \phi] \rightarrow[(\chi \rightarrow \psi) \rightarrow(\chi \rightarrow[\phi \wedge \psi])]$
6. $\phi \rightarrow(\phi \vee \psi)$, o $\rho \rightarrow(\phi \vee \psi)$
7. $[\phi \rightarrow \chi] \rightarrow[(\psi \rightarrow \chi) \rightarrow([\phi \vee \psi] \rightarrow \chi)]$

The preceding is an axiom system for SC [5]. In other words, the axioms of SC when translated are elements of STIMA.

I will now show that the collection STIM $_{A}$ and the collection of provable propositions of SC (PROV) are identical under the translation described. Since SC is complete, this will demonstrate that PROV $=\mathrm{STIM}_{\mathrm{A}}=\mathrm{TAUT}$, where TAUT is the collection of tautologies of SC. In conjunction with the preceding Lemmas, this will demonstrate that the calculus of stimuli is equivalent to SC.

\section{Theorem 1: $\quad \mathrm{PROV} \supseteq \mathrm{STIMA}$}

pf: It must be shown that any stimulus that reduces to A translates to a provable formula of SC.

a. the stimulus may translate to an axiom of SC in which case the proof is complete.

b. if the stimulus, s, does not translate to an axiom of $\mathrm{SC}$, then let the formula it translates to be fs. Let $\mathrm{R}$ be any realization of SC. $R\left(f_{A}\right)=\mathbf{1}$, and since s reduces to $A$, by

Lemma 3: $\mathrm{R}\left(\mathrm{f}_{\mathrm{s}}\right)=\mathbf{1}$. That is, fs $\in$ TAUT. But SC is complete, so

TAUT $=$ PROV . Therefore, $\mathrm{fs} \in \mathrm{PROV}$, and

PROV $\supseteq S T I M A$.

Theorem 2: STIMA $\supseteq$ PROV

pf. It must be shown that any provable formula of SC translates to a stimulus that reduces to $\mathrm{A}$. This will be proved by induction on the length of proof, i.e., either the provable formula is an axiom of SC, or if $\mathrm{f}$ translates to a stimulus, s; translates to a stimulus, s'; and g, translates to a stimulus, t; and both $\mathrm{s}$ and $\mathrm{s}$ ' reduce to $\mathrm{A}$, then $\mathrm{t}$ also must reduce to $\mathrm{A}$.

a. all axioms when translated to a stimulus reduce to A as shown in the seven examples of the first section.

b. $\mathrm{f}$ translates to s reduces to A and g translates to $\mathrm{t}$. Since

$\mathrm{f} \rightarrow \mathrm{g}$ reduces to $\mathrm{A},[\mathrm{s}] \mathrm{t}=\mathrm{A}$. But $\mathrm{s}$ reduces to $\mathrm{A}$ so $[\mathrm{A}] \mathrm{t}=\mathrm{A}$. 
$[\mathrm{A}]=\emptyset$, so $\emptyset \mathrm{t}=\mathrm{A}$ and therefore, $\mathrm{t}=\mathrm{A}$, and STIMA $\supseteq$ PROV

Theorem 3: $\quad$ PROV $=$ STIMA $=$ TAUT

pf: $\mathrm{PROV}=\mathrm{TAUT}$ by completeness of SC. Theorems 1 and 2

indicate STIMA = PROV. Therefore,

PROV = STIMA $=$ TAUT.

The preceding accounts for all of the necessary preliminaries for one result of this development.

Theorem 4: The calculus of stimuli is equivalent to SC

pf. Interpret the calculus of stimuli as described and apply Lemma 1 and Theorem 3.

Though this assures the equivalence of SC and the calculus of stimuli, it does not imply that the inductive constructsincluding the natural sciences-derived from sensory data necessarily retain this logical bias. It is this latter fact that ensures the commonalty of logic underlying the models of the external universe created by organisms satisfying very weak topological constraints.

\section{An astrobiological theorem}

This proof is based on two axioms: 1. the external world can be known only as an internal model, and 2. each stage of model-building is inductive-i.e., the process of increasing complexity is successive identification of equivalence. The first stage of internal reconstruction of the external ambience involves the identification of separate objects within the global patterns of activation. It first must be shown that objects, as equivalence classes of subsets of the global patterns of activation, retain the logical bias of the calculus of stimuli. It then must be proven that any further inductions derived from objects preserve the calculus of stimuli.

Theorem 5: Any internal inductive construct derived from sensory data preserves the calculus of stimuli.

pf. Proof is by induction on the length of the inductive chain.

Let $\Sigma_{1}, \ldots, \Sigma_{n}$ represent separate patterns of global activation of the same organism so that they represent different activations of the same set of transducing units, $A^{*}$. Let $a_{1} \subseteq \Sigma_{1}$, $\ldots, a_{n} \subseteq \sum_{n}$ be " $n$ " stimuli that are identified as representing the same object. I.e., $a_{1} \approx_{i} \ldots \approx_{i} a_{n}$ where " $\approx_{i}$ " means equivalent under the induction "i." By definition, the equivalence relation " $\tilde{i}_{i}$ " must contain all elements that are shared by $a_{1}, \ldots, a_{n}$ i.e., the conjunction of $a_{1}, \ldots, a_{n}$. It must be shown that if $a_{1}, \ldots$, $a_{n}$ are described by the calculus of stimuli, then so is $\left[\left[a_{1}\right] \ldots\right.$ $\left.\left[a_{n}\right]\right]$. The stimulus $\left[\left[a_{1}\right] \ldots\left[a_{n}\right]\right]$ is a pattern of activation of $A^{*}$. Since $a_{m} \subseteq \sum_{m}$ of $A^{*}$ is a stimulus for $m=1, \ldots, n$, successive applications of rules 2 and 3 imply $\left[\left[a_{1}\right] \ldots\left[a_{n}\right]\right.$ is a stimulus. By above theorems and lemmas, the equivalence class induced by " $\tilde{\sim}_{i}$ " preserves the calculus of stimuli.

Let $\mathrm{P}_{1}, \ldots, \mathrm{P}_{\mathrm{r}}$ be equivalence classes obtained by a sequence

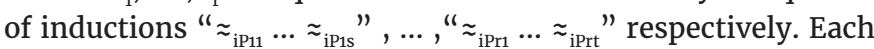
$\eta_{\mathrm{m}} \in \mathrm{P}_{\mathrm{m}}$ for $\mathrm{m}=1, \ldots, \mathrm{r}$ is of the form $\left(\mathrm{a}_{1} \tilde{\sim}_{\mathrm{iP11}} \ldots \approx_{\mathrm{iP1s}} \eta_{1}\right), \ldots,\left(\mathrm{a}_{\mathrm{r}} \tilde{\mathrm{i}}_{\mathrm{iPr} 1} \ldots\right.$ $\tilde{z}_{\text {iPrt }} \eta_{\mathrm{r}}$ ) for some patterns of activation of the transducing units, $\mathrm{a}_{1}, \ldots, \mathrm{a}_{\mathrm{r}}$. The calculus of stimuli is retained in $\mathrm{P}_{1}, \ldots, \mathrm{P}_{\mathrm{r}}$ by assumption and, therefore, $\eta_{1}, \ldots, \eta_{\mathrm{r}}$ are valid stimuli. Let $R$ be an equivalence class of $\mathrm{P}_{1}, \ldots, \mathrm{P}_{\mathrm{r}}$ under some equivalence relation " $\approx$ ", then it must be shown that the calculus of stimuli is retained in R. Now $\mathrm{R}$ must contain some shared elements of $\mathrm{P}_{1}, \ldots, \mathrm{P}_{\mathrm{r}}$ under " $\approx$ ", i.e., for some $\zeta_{1} \in \mathrm{P}_{1}, \ldots, \zeta_{\mathrm{r}} \in \mathrm{P}_{\mathrm{r}}$ with $\zeta_{\mathrm{k}} \subseteq$ $\eta_{\mathrm{k}},\left[\left[\zeta_{1}\right] \ldots\left[\zeta_{\mathrm{r}}\right]\right]$ must be shown to be a stimulus. By successive applications of rules 2 and 3 this must be so since all $\eta_{1}, \ldots$, $\eta_{\mathrm{r}}$ are stimuli (by assumption) and therefore so are $\zeta_{1}, \ldots, \zeta_{\mathrm{r}}$. By preceding theorems and lemmas, the equivalence class $R$ preserves the calculus of stimuli.

The essence of this theorem is that all objects, relationships, and interactions forming the internal model of the external universe constructed from sensory data contain an encoded logic resulting from the necessity of transduction at the boundary surface. Therefore, the laws discovered governing the models of the external world-themselves being inductive constructs - also contain this logical bias.

Theorem 6: Any sufficiently advanced organism of nonzero, finite volume (with boundary) has embedded in its model of the world a logical system equivalent to SC.

pf. Let $A^{*}$ be the set of transducing units of an arbitrary organism. Because an organism of non-zero finite volume (with boundary) must convert the external milieu to an internal one, $A^{*}$ is not empty. Interpret the calculus of stimuli as described, and apply Theorem 4 and 5 .

The astrobiological theorem demonstrates that there is a logical bias embedded in the most elementary unit of sensation and that this bias is faithfully transmitted to any internal inductive construct originating in sensory transduction. By the theorem's axioms, all that can be known of the external world is such an internal model, and this model is inductively created. Therefore, SC forms the 'natural' logic in the model of the world of any organism with a boundary surface. This fact has consequences for a variety of scientific issues and the following comments illustrate different perspectives on some classic problems in mathematics and physics.

\section{Constructive and formal mathematics}

The relationship between structure and logic identified in the theorem suggests a synthesis of mutually exclusive ideas about the nature of mathematics. For example, the theorem retains the universal nature of mathematical forms found in formal or platonic theories. However, universality is not due to independent external existence of these forms, as platonists generally believe, but rather to a universal necessity to convert the external world to an internal one. Conversely, an epistemological origin of mathematics is confirmed, supporting constructive notions, but this is not a purely human limitation, as the constructivists imply, since it applies to any physical organism. Finally, intuitionist philosophies contend that logic is derived from experiential, finitary mathematics and is not reliably applied to infinitary cases. This results in the distrust of indirect proofs and such logical principles as the excluded middle. The theorem again suggests areas of agreement and disagreement with this philosophy. Contrary to the intuitionist view, logic is synthetic a priori relative to mathematics since it 
arises from the physical structures of sensation. However, it is exactly this origin in the objects of perception that would seem to limit its reliable utility to the finitary perceptible universe, in agreement with the intuitionist viewpoint.

In summary, an attractive aspect of the astrobiological theorem is the synthesis it offers for several disparate ideas about the nature of mathematics. Perspectives that were mutually exclusive are shown to arise as simply a difference in emphasis within a more general structure.

\section{Utility of mathematics in physics}

Eugene Wigner (1902-1995) eloquently expressed the perplexity engendered by the apparently fortuitous utility of mathematics in physics [6]. This has become even more apparent in string theories in which, apparently, the only reality is the mathematics. The astrobiological theorem may suggest, at least phenomenologically, why this relationship exists. Since a logical system is embedded in the most elementary form of perception, any inductive structure internally reconstructed from these elementary percepts, including physics, is subject to this initial constraint. However, this same constraint, being metamathematical, limits the (natural) deductive forms of mathematics. This, of course, does not impy that other logical systems cannot be conceived and investigated, as obviously is the case, but rather that SC is, in a sense, a 'natural' logic that is a consequence of physical structure. In this interpretation, the utility of mathematics in physics is due to a similar logic embedded at the first stage of perception. Physics, which describes the external world, and mathematics, which some believe is a human creation, are coherent because both contain a logic whose origin is a consequence of physical, topological structure.

\section{Quantum mechanics}

The mathematical description of non-relativistic quantum mechanics was completed in 1925 superseding the collection of empirical observations and ad hoc assumptions known as the old quantum theory [7-9]. Upon completion of this project it was realized that the formal structure contained significant interpretive problems. The explanation of these difficulties engaged the greatest scientific minds of the previous century, without satisfactory resolution [10-12].

The Copenhagen interpretation of quantum mechanics [8], supported by experimental violation of Bell's inequalities [13], implies that there is a fundamental interaction between perception and reality as manifested, for example, in measurement systems, non-local causality, and the reality of unobserved quantum processes. However, this explanation is verified only by the phenomena it is supposed to explain - there is no independent evidence that such an interaction is possible. Of course, it is not essential that other similar processes be known. On the other hand, the astrobiological theorem does provide independent evidence supporting the possibility of such an interaction. By explicitly demonstrating a logical bias introduced by physical structure, it provides a particular example of a constraint on observable reality imposed by perception.

\section{Conclusion}

An abstract description of the process of sensation is shown to be equivalent to the logic of sentences. In this way, SC can be considered a consequence of physical structure. This logical bias is faithfully preserved in any internal inductive constructs based on sensory data, and therefore, any sufficiently advanced physical organism will share a form of SC. This proves a theorem about extraterrestrial intelligence. In addition, the formal consequences identified by the theorem suggests new interpretations and, therefore, new areas of research in physics and mathematics.

\section{References}

1. Shannon CE (1938) A symbolic analysis of relay and switching circuits Electrical Engineering 57: 713-723. Link: https://bit.ly/2SZXYlu

2. Steinberg DA (2014) The origin of scientific neurology and its consequences for modern and future neuroscience. Brain 137: 294-300. Link: https://bit.ly/3dvliji

3. Steinberg DA (1997) In Brain, mind and physics, edited by P Pylkkanen, P Pylkko and A Hautamaki (IOS Press, Amsterdam, 1997). Link: https://bit.ly/350NbNq

4. Steinberg DA (1999) A short history of Neurology: The British contribution 1660-1910, edited by F. Clifford Rose (Butterworth-Heinemann, Oxford, 1999). Link: https://bit.ly/3nZNssS

5. Bell JJ, Slomson AB (1971) Models and Ultraproducts: An Introduction (North Holland Publishing Co., London, 1971) 36-37.

6. Wigner E (1960) The Unreasonable Effectiveness of Mathematics in the Natural Sciences. Comm. Communications on Pure and Applied Mathematics 13: 1-14. Link: https://bit.ly/3m4p3k7

7. Dirac PAM (1926) Quantum mechanics and a preliminary investigation of the hydrogen atom. Proceedings of the Royal Society 109A. Link: https://bit.ly/2H2OX8Q

8. Heisenberg W (1968) Sources of Quantum Mechanics, edited by BL van de Waerden (Dover Publications Inc., New York, 1968)

9. Schrodinger E (1926) Quantizierung als Eigenwertproblem (Vierte Mitteilung) / Quantization as a Problem of Proper Values. Part IV. Annalen der Physik 81 109. Link: https://bit.ly/31f471D

10. Bohr N (1928) The Quantum Postulate and the Recent Development of Atomic Theory ${ }^{1}$. N Nature 121: 580-590. Link: https://go.nature.com/2T5xD5j

11. Einstein A (1991) Autobiographical. Notes translated by P.A. Schilpp (Open Court Press, La Salle, Illinois, 1991). Link: https://bit.ly/2SZ8c5A

12. Einstein A, Podolsky B, Rosen N (1935) Can Quantum-Mechanical Description of Physical Reality Be Considered Complete?. Phy Rev 47. Link: https://bit.ly/2T00tnS

13. Aspect A, Dalibard J, Roger G (1982) Experimental Test of Bell's Inequalities Using Time-Varying Analyzers. Phys Rev Lett 49. Link: https://bit.ly/3duQcJE 